\title{
Innovative IoT Sensing and Communication Unit in Agriculture
}

\author{
David Krčmařík ${ }^{1 *}$, Michal Petrů${ }^{1}$, Reza Moezzi $^{1,2}$ \\ ${ }^{1}$ Institute for Nanomaterials, Advanced Technologies and Innovation, Technical University of Liberec, Studentská 2, Liberec, \\ Czech Republic \\ ${ }^{2}$ Faculty of Mechatronics, Informatics and Interdisciplinary Studies, Technical University of Liberec, Studentská 2, Liberec, \\ Czech Republic
}

Corresponding Author Email: david.krcmarik@tul.cz

https://doi.org/10.18280/ejee.210302

Received: 6 March 2019

Accepted: 28 April 2019

\section{Keywords:}

Internet of thing, smart agriculture, tensometer, precision agriculture, GSM, big data

\begin{abstract}
This paper aims to propose an innovative reconfigurable sensing unit, suited to be used in harsh agriculture environment. The heart of the unit is a PCB with Linux based processor which is established such a way that the unit communicates via a backend, SQL based database with a user-friendly web interface. It can aggregate several data sources: tensometers, accelerometer, temperature sensor, CAN, digital and analog inputs. It communicates via GSM and It implements GPS information to provide precise real-time position. The unit is designed to be powered with different power sources. Collection the units which are used, can be easily reached using standard private network. The backend enables the users to configure desired actions. One can choose certain data to be sensed. It is possible to configure also the data acquisition rate and time interval between two packages sent to backend. Data are available through the web interface and can be downloaded to universal format of CSV for further processing. It has been shown that the whole system is capable to monitor user defined data limits and if such a limit is reached a notification (e.g. SMS) can be sent to a predefined destination.
\end{abstract}

\section{INTRODUCTION}

Precision agriculture (PA) is a hot topic in recent years. It spans from robots, drones, artificial intelligence down to IoT (internet of things) sensors and actuator networks. Precision agriculture enables to maximize the production with respect to the same amount of inputs and at the same time preserving the resources. This paper would focus on the IoT part of precision agriculture.

Modern precision agriculture is vastly dependent on a huge amount of collected data. Data acquisition is possible via popular IoT networks. IoT enabled device, needs to have some kind of sensor and a way of sending the information to backend. IoT devices are often located in remote areas where cable connection is not feasible. There are many networks which can be used for communication: WiLD, SigFox, LoRa, GSM, Zigbee [1] or any integrated wireless network [2]. For example, in the rural regions for PA with low cost, The WiFibased Long Distance (WiLD) network is used to connect IoT devices for communication [3-5]. But the WiFi based network has its disadvantages according to its distance coverage. One of the most used one is GSM data (GPRS or 3G) since such networks are available nearly everywhere and are widespread. One must take into account also energy-efficiency. The best scenario is when the IoT devices are capable to self-charge using solar power, residual energy generated by the device, etc. The data which came from different devices suffer from inconsistency of protocols, time delays, etc. So, there must be some management system gathering data - Node-Red (for user-friendly small-scale networks) or some specialized system like SmartFarmNet [6].
The trend in using IoT based solutions for agriculture is clearly apparent already many years. Among such solutions mainly monitoring of various things (water, humidity, air flow, concentration, etc.) is a most common scenario [7]. Controlling some actuators via IoT is still evolving. The different algorithms can be applied in control theory such as fuzzy $[8,9]$ or implementation of conventional PID as described in [10, 11]. Hlava et al. [12] also investigated on hybrid processes. In agricultural purposes, regardless of control theory in deep view, the most common case is controlling smart greenhouses [13]. It is partly due to the fact, that for controlling unlike monitoring it is needed usually more energy and resources. We also must consider processing of great numbers of data. Such field is labeled as big data and several platforms exist (e.g. Azure).

There are many efforts to use IoT based solutions for monitoring agriculture related data. In [14] Popovic and his colleagues have proposed an experimental platform for use in aquaculture monitoring, irrigation (control scenario) and ecological monitoring. They made their solution based on cheap and widely available Arduino and Raspberry Pi with a family of sensor products from Libelium. They predicted a future in usage of standard protocols (MQTT, CoAP) and exploitation of widely spread languages like Python or statistical R. Various case studies used in real scenarios are presented in [15]. The precision agriculture is presented as a network which uses standardized protocols where the nodes are to a certain degree intelligent self-configurable upon the environmental conditions. Currently, most of the real-world scenarios are from such areas: irrigation control, pest and disease control, cattle movement, water quality monitoring, 
control and monitoring of greenhouses, using drones for intelligent field assessment. In [16] the authors present also a nice list of IoT ready sensors and control nodes. The information is sent to a central hub using wireless technologies, and the backend can be either built from scratch or one can use existing infrastructure which in certain cases is for free (ThingSpeak, Xively, Plotly). The acquired data are necessary to process and evaluate. Here comes into play so-called Big Data infrastructure. Concerning Big Data and data analytics, a thorough search is available in [16]. Several such agriculture management information systems are available (Easyfarm, Farmlogs, Cropx, OnFarm, Farmx, etc.). Data analytics is nowadays one of the key concepts which can help with decision making and prediction from vast amount of data. From acquired data, useful analysis can be made to perform the desired plan. Such data can be also supplemented with camera images [14].

In this practical paper, we have proposed an innovative $\mathrm{PCB}$ unit in the domain of IoT, connecting with various agricultural and farming devices to monitor and transferring information based on GSM data services.

This paper, is structured into six sections. The IoT system and charging of monitoring unit are discussed in the second section. Section third, presents the practical tested sensors and communication unit. Forth section dives into the backend which implements MSSQL database architecture. The Big data would be discoursed in the fifth part and Finally, last section concludes the paper.

\section{CHARGING OF MONITORING UNIT}

The methodology is focused on an autonomous measuring unit which is used with tractors to monitor accelerometers, tensometers, CAN interface, analog and digital inputs along with GPS information. This is useful for monitoring the tractor operation (whether it works according to optimal usage). Such units have an energy management and three sources of internal battery charging. The charging structure in a microscopic means can be found in [17]. Battery management is an import key in design of the unit since the unit should be independent from the tractor. It can be charged using a solar panel, a generator unit which is mounted on a rotational part of the machine and directly from the battery of the tractor. The unit has internal battery block which enables seamless functioning even for a certain time even when there is no any energy source (Figure 1). The regression analyses [18] which is an analytical model can be used to predict the efficiency of power usage. the In Figure 1 is also visible the solar panel which is flexible and can be mounted directly on the top of the box or somewhere else. We have analyzed the possible usable solar energy during the year. For central Europe (Czech Republic) the graph showing utilized photovoltaic gains is in Figure 2. There is a significant increase in energy when using MPPT (maximum power point tracking) regulator. The minimum amount of energy is reached in January - around $20-25 \mathrm{kWh} / \mathrm{m}^{2}$. If we consider this minimum value and decrease it little bit (due to suboptimal orientation angle, possible dust) to value 15 $\mathrm{kWh} / \mathrm{m}^{2}$ the chosen solar panel (SO28 v.1) with surface $0.07 \mathrm{~m}^{2}$ should give us in January about $1 \mathrm{kWh}$. The overall consumption of the electronics and related communication unit is less than the available power from a solar panel.

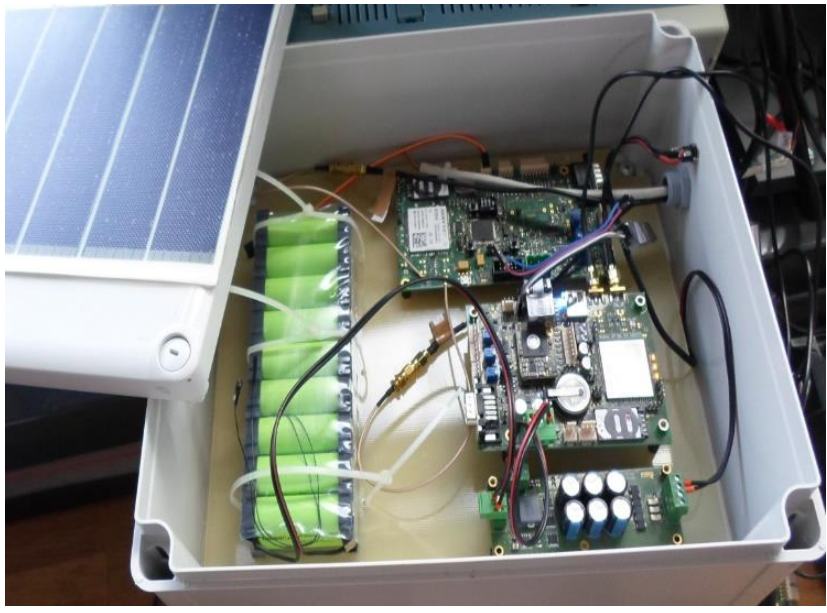

Figure 1. A box including a battery block (on the left), charging management unit (bottom right) and sensor and communication unit (right)

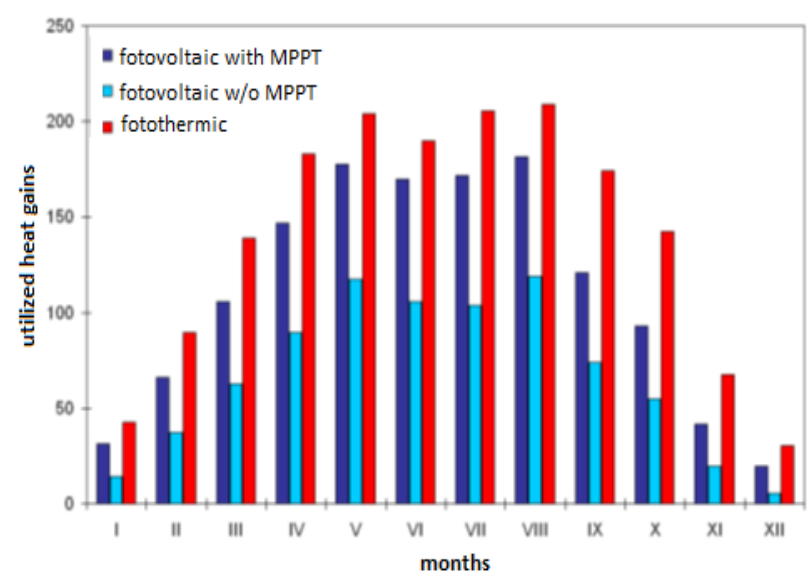

Figure 2. Graph showing utilized heat gains during the year (MPPT - solar charging regulator)
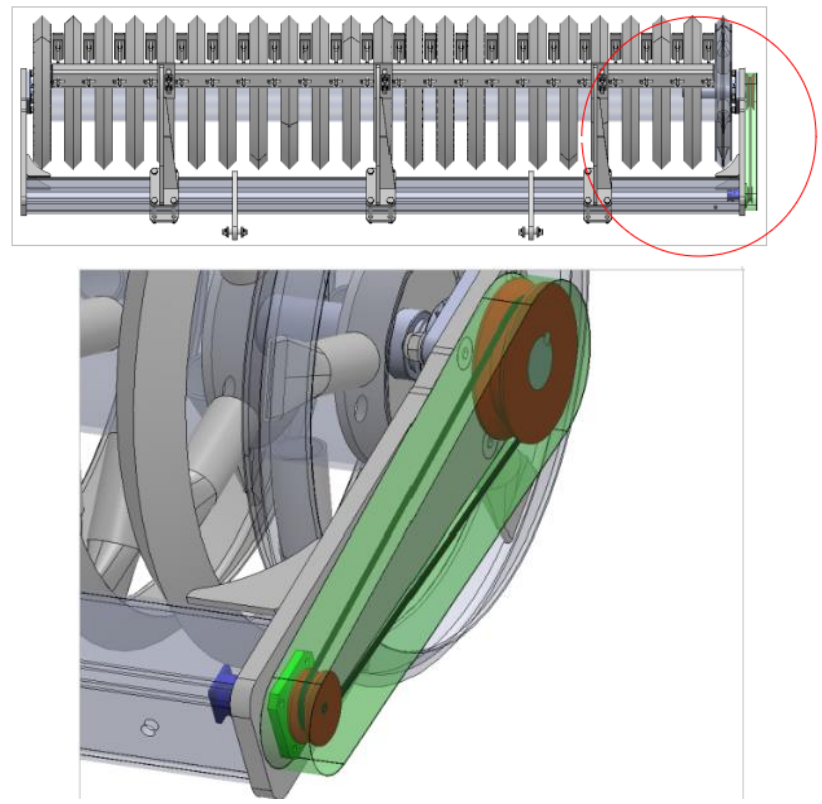

Figure 3. Rotational part of suspension machinery and detail of mounting (below) 


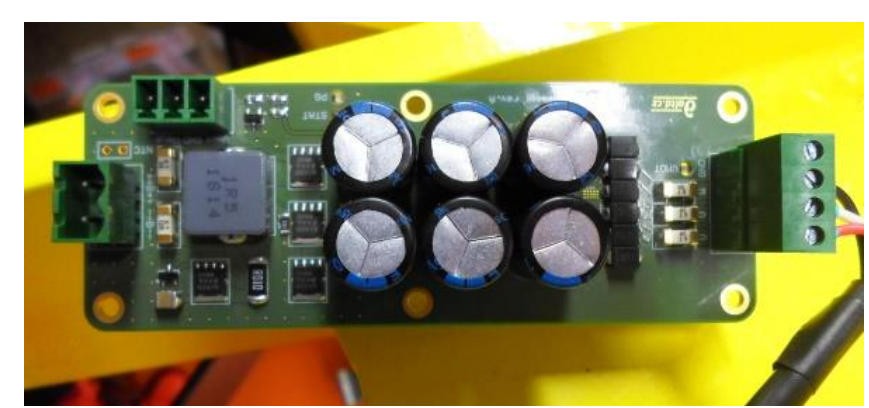

Figure 4. Power management unit

The unit is mounted on suspension agriculture machinery (tillage, seeding, fertilizing, crop management residue, etc.). The frame used for the components is built by carbon fiber compound which has shown a realiable solution for special applications [19]. The reliability of the energy charging can be substantially increased when a generator mounted within some rotational part of suspension technique is used. In Figure 3 there is a schematic of mounting. We have used a cheap compact generator (NEMA17 mount) with a gearbox which is giving us appropriate voltage around $24 \mathrm{~V}$ when the tractor is moving $15 \mathrm{~km} / \mathrm{h}$. We have measured the charging current for a sweep of tractor speeds. For that we have used a specially designed charging management unit. The generator starts to give minimal power at the speed of $5 \mathrm{~km} / \mathrm{h}$ and reaches its maximum current with $8 \mathrm{~km} / \mathrm{h}$ speed. It maintains the maximum current up to cca $40 \mathrm{~km} / \mathrm{h}$. After this speed is exceeded the power management unit (Figure 4) blocks the excessive power not to damage the block of battery cells. From test field we know that normal speed of agriculture mechanization in the field is within $10-20 \mathrm{~km} / \mathrm{h}$.

\section{SENSOR AND COMMUNICATION UNIT}

We have designed a 4-layer PCB which has GSM/GPS module (Quectel EC25-E) and a processor Atmel SMART SAMA5D3 (ARM Cortex-A5 @536MHz) running a Linux distribution Myrmica 2.0 (Figure 5). It has several connectors which are necessary for agriculture usage: Three tensometers, embedded accelerometer, temperature sensor, analog pins (0$12 \mathrm{~V}$ ), digital and tamper pins, CAN, USB connection.

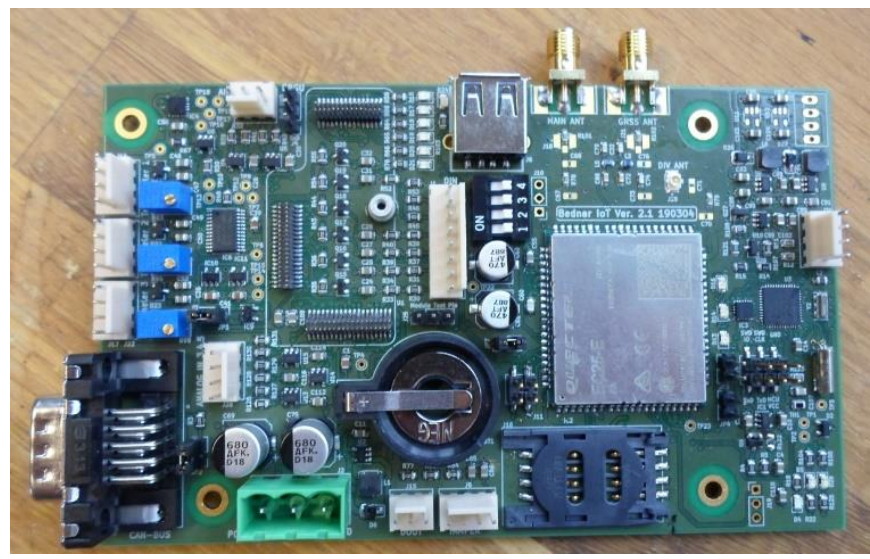

Figure 5. 4-layer sensor and communication unit

In order to acquire data from accelerometer and tensometers we need to sample the data more frequently than other sensors. We have measured typical tensometer profile of data (Figure 6) and figured out that in order to detect the peaks we need at least sampling frequency of $50 \mathrm{~Hz}$.

For calibration purposes we have designed a test tensometer preparation (Figure 7).

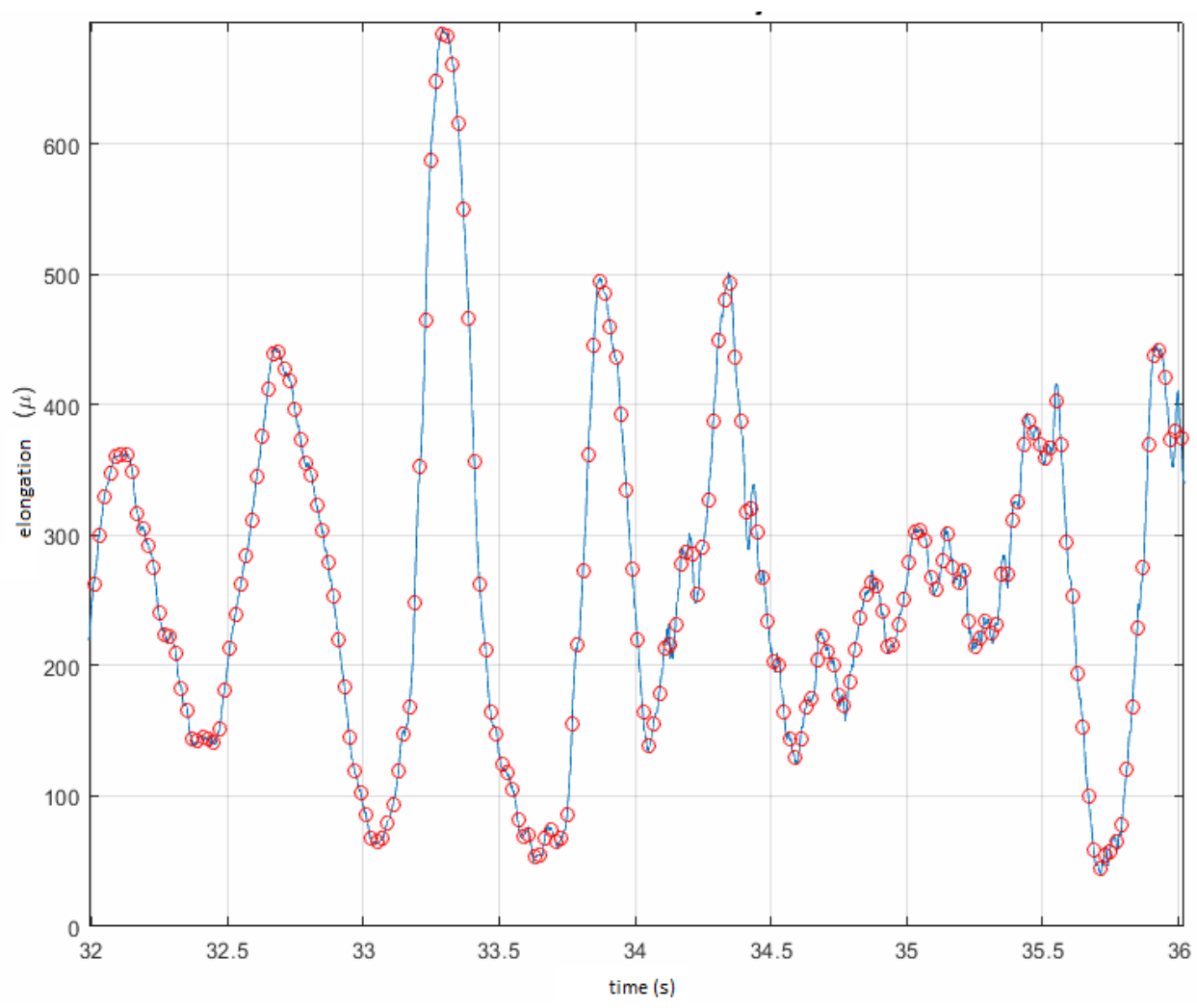

Figure 6. Tensometer measurement with apparent peak and sampling points 


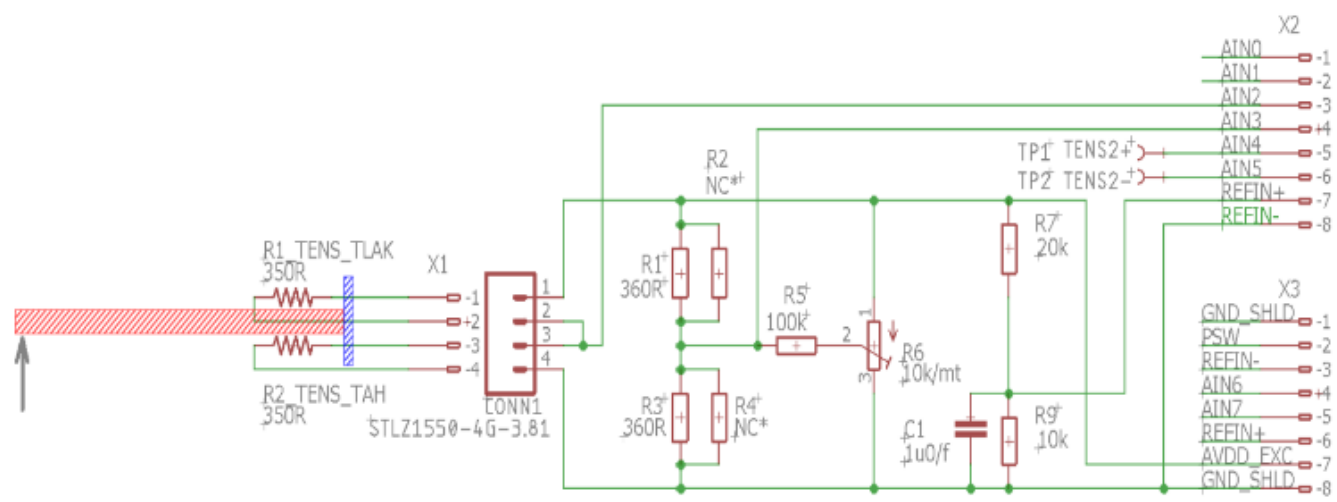

(a) Scheme of tensometer

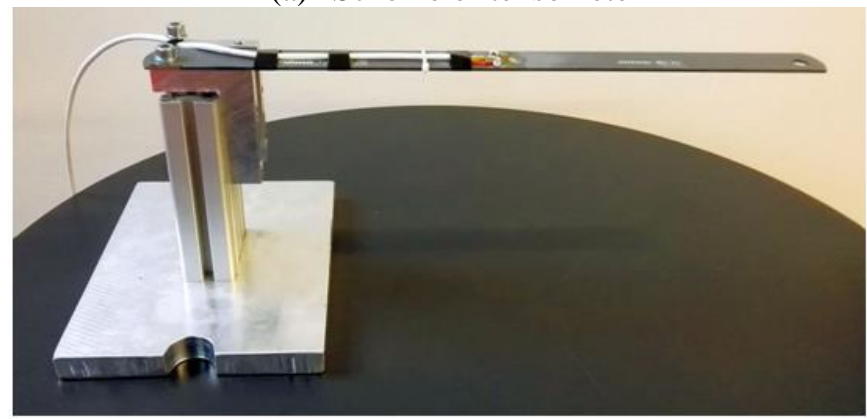

(b) Physical tensometer

Figure 7. Tensometer preparation

\section{BACKEND}

Data are sent using standard TCP/IP over GSM connection to a backend. Backend consist from an MSSQL server where raw data are stored. This interface is available through a web service. A user interface consists of web pages (frontend) where all necessary information and possible configuration of units is done. There are two groups of users: administrators and common users. Administrators can create new configurations and create new units. They can then assign a specific configuration to a specific unit. Administrator can also assign certain common users to be able to see and download data about a particular unit. The web site runs on the address http://gsmmachine.bednarfmt.com:8080. In configuration an administrator can choose which information, should be sent from unit to backend. He can also choose the IDC (interval for data collections) and ISE (interval for data sending). E.g. when IDC is 5 and ISE is 600 it means that data are scanned every 5 second and after 10 minutes data are aggregated into one JSON POST and send it to a web service endpoint. It is also possible to configure the APN name, SMS number for special purposes and configuration of wakening from sleeping mode (AWK).

Sleeping modes are very important things for IoT units. When the suspension agriculture mechanism is not in work the unit should go to the sleep mode in order to save energy. We have prepared the unit for 3 scenarios. Such scenarios are possible due to a small ST processor with very low energy consumption $(<1 \mu \mathrm{W})$. The unit is constantly monitoring acceleration and generator voltage; it has its own real time clock and the GSM module is always available for receiving SMS. One can choose whether the unit starts to work upon a certain acceleration or generator voltage is reached or after some predefined time of inactivity or using a wakening SMS.
This is configured as a string into the AWK parameter. For example, the string "accel $2 \mathrm{G} 20 \mathrm{~min}$ " means that the unit awakens when an acceleration of at least $2 \mathrm{G}$ is sensed on either axis and goes to sleep when no acceleration is measured at least for 20 minutes.

The user can visualize all the measured and sent data using the web interface (Figure 8). He can choose a timespan in which he is interested. He can also export the data into a CSV file and post process it later.

\section{BIG DATA}

The main purpose of data collection through our IoT unit is to gather enough data for further "artificial intelligence" processing. Behind the backend a big data engine is running and evaluating the measured data. Basically, this is necessary since the amount of data is increasing and there has to be a backend with large amount of RAM and processing power to compare various measured quantities and correlate them. We are experimenting with Apache Spark and Elasticsearch. We found that Elasticsearch is good for finding certain occurrences of events within our data whereas Spark can be probably more valuable in terms of finding some unexpected correlations. In order to get some valuable results, it is necessary to collect the data over larger time span. When some data diverts significantly and at the same time the suspension agriculture mechanism is reported to be broken such an incident is entered into the system. The more of such incidents happens the more the whole machine learning model can be particularly trained for predicting certain scenarios and hence increase the reliability of the devices. A nice library MLlib can be used to warn before situations which were trained before. 


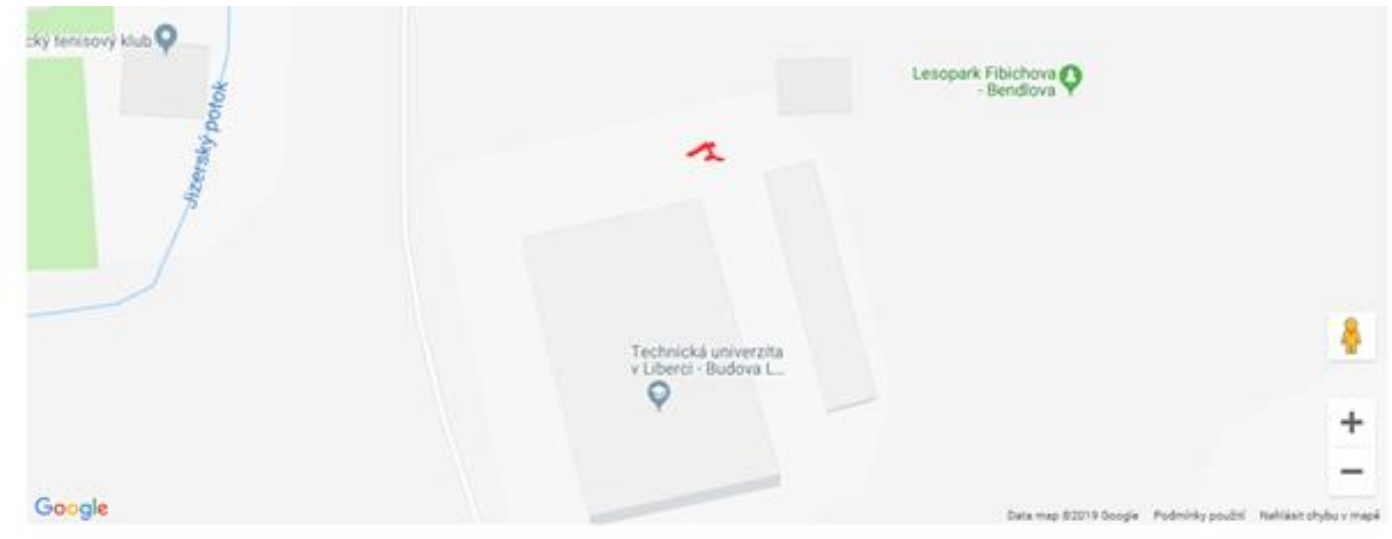

diBinarylnputs_1

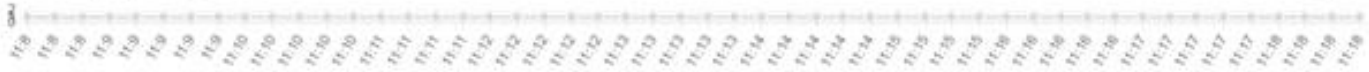

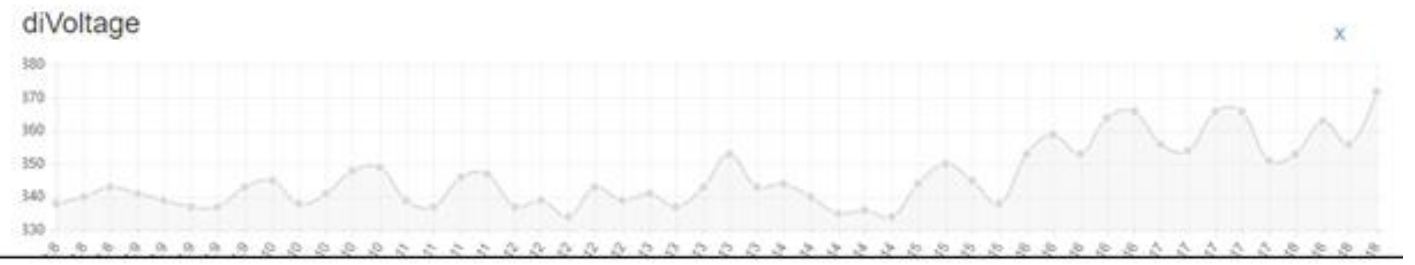

diAccelerometer

31

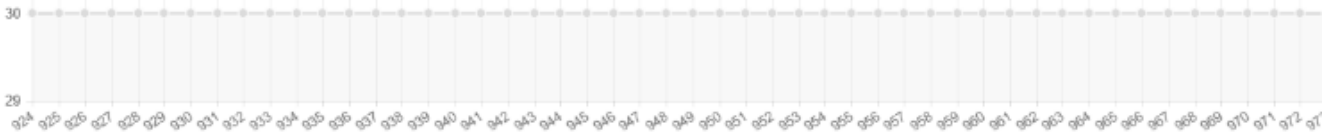

diBinarylnputs_1

105

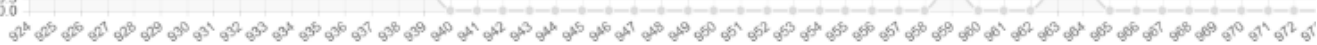

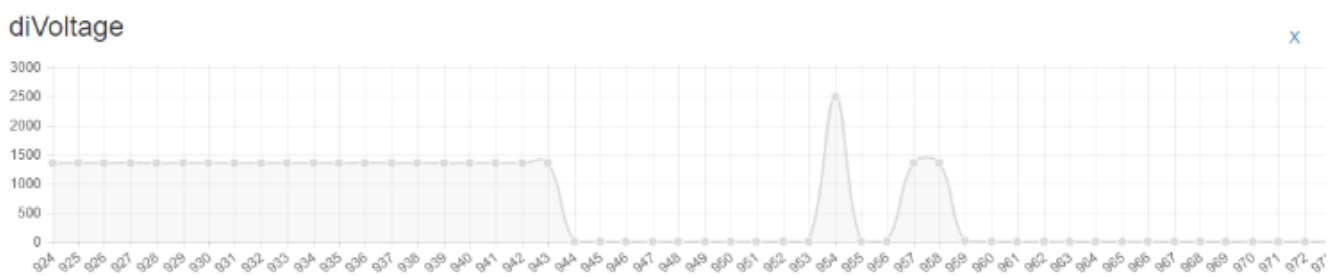

Figure 8. Web interface showing the movement of the unit within the map (above) and chosen data in graphs

\section{CONCLUSIONS}

We have presented an IoT unit which can be used in a harsh environment of agriculture. It is meant to be used for monitoring the suspension agriculture mechanisms. It has its own battery and several ways how to recharge the battery. Each unit is addressable via a web interface and can be configured independently. All the information from all the units in the field are collected into one MSSQL database where the data can be retrieved for further processing, visualization or export. We believe that the Apache Spark combined with the MLlib library can be a useful tool in order to diagnose unwanted behavior and predict fault detection.as future study, the IOT system in smart agriculture as described in [20] is under investigation and the group has predicted innovative position tracking with Augmented Reality technology [21] for position tracking which is capable to link AR technology with precision agriculture.

\section{ACKNOWLEDGMENT}

The result was obtained through the financial support of the Ministry of Education, Youth and Sports of the Czech Republic and the European Union (European Structural and Investment Funds - Operational Programme Research, 
Development and Education) in the frames of the project "Modular platform for autonomous chassis of specialized electric vehicles for freight and equipment transportation", Reg. No. CZ.02.1.01/0.0/0.0/16_025/0007293.

\section{REFERENCES}

[1] Jawad, H.M., Nordin, R., Gharghan, S.K., Jaward, A.M., Iamail, M. (2017). Energy-efficient wireless sensor networks for precision agriculture: A review. Sensors, 17(8): 1781. https://doi.org/10.3390/s17081781

[2] Tamre, M., Hudjakov, R., Shvarts, D., Polder, A., Hiiemaa, M., Juurma, M. (2018). Implementation of integrated wireless network and MATLAB system to control autonomous mobile robot. International Journal of Innovative Technology and Interdisciplinary Sciences, 1(1): $18-25$. https://doi.org/10.15157/IJITIS.2018.1.1.18-25

[3] Bhagwat, P., Raman, B., Sanghi, D. (2014). Turning 802.11 inside-out. ACM SIGCOMM Computer Communication Review, 34(1): 33-38. https://doi.org/10.1145/972374.972381

[4] Chebrolu, K., Raman, B. (2007). FRACTEL: A fresh perspective on (rural) mesh networks. Workshop on Networked Systems for Developing Regions, pp. 8:1-8:6. https://doi.org/10.1145/1326571.1326583

[5] Hussain, M.I., Ahmed, Z.I., Sarma, N., Saikia, D. (2016). An efficient TDMA MAC protocol for multi-hop WiFibased long distance networks. Wireless Personal Communications, $\quad$ 86(4): 1971-1994. https://doi.org/10.1007/s11277-015-3165-9

[6] Jayaraman, P.P., Yavari, A., Georgakopoulos, D., Morshed, A., Zaslavsky, A. (2016). Internet of Things platform for smart farming: Experiences and lessons $\begin{array}{lll}\text { learnt. } & \text { Sensors, } & 1684\end{array}$ https://doi.org/10.3390/s16111884

[7] Talavera, J.M., Tobon, L.E., Gomez, J.A., Culman, M.A., Aranda, J.M., Parra, D.T., Quiroz, L.A., Hoyos, A., Garreta, L.E. (2017). Review of IoT applications in agroindustrial and environmental fields. Computers and Electronics in Agriculture, 142: 283-297. https://doi.org/10.1016/j.compag.2017.09.015

[8] Reza, M., Minh, V.T. (2016). Fuzzy logic control for non-linear model of the ball and beam system. The Proceedings of the International Conference of DAAAM Baltic "Industrial Engineering", 2016-April 60-65.

[9] Minh, V.T., Mart, T., Moezzi, R., Oliver, M., Martin, J., Ahti, P., Leo, T., Mart, J. (2016). Performances of PID and different fuzzy methods for controlling a ball on beam. Open Engineering, 6(1): 145-151. https://doi.org/10.1515/eng-2016-0018

[10] Hlava, J., Zemtsov, N., Frantsuzova, G. (2016). Application of PID controller based on the localization method for ancillary service provision. 2016 International Siberian Conference on Control and
Communications, SIBCON 2016 - Proceedings. https://doi.org/10.1109/SIBCON.2016.7491747

[11] Hlava, J. (2009). Hybrid modelling and control of a power plant three-stage reheater. IFAC Proceedings Volumes, 42(17): 32-37. https://doi.org/10.3182/20090916-3-ES-3003.00007

[12] Hlava, J., Sulc, B. (2008). Advanced modelling and control using a laboratory plant with hybrid processes. IFAC Proceedings Volumes, 41(2): 14636-14641. https://doi.org/10.3182/20080706-5-KR-1001.02479

[13] Tzounis, A., Katsoulas, N., Bartzanas, T., Kitta, C. (2017). Internet of things in agriculture, recent advances and future challenges. Biosystems Engineering, 164: 3148. https://doi.org/10.1016/j.biosystemseng.2017.09.007

[14] Popović, T., Latinović, N., Pešić, A., Zečević, Ž., Krstajić, B., Djukanović, S. (2017). Architecting an IoTenabled platform for precision agriculture and ecological monitoring: A case study. Computers and Electronics in Agriculture, 140: 255-265. https://doi.org/10.1016/j.compag.2017.06.008

[15] Ray, P.P. (2017). Internet of things for smart agriculture: Technologies, practices and future direction. Journal of Ambient Intelligence and Smart Environments, 9(4): 395-420. https://doi.org/10.3233/AIS-170440

[16] Elijah, O., Rahman, T.A., Orikumhi, I., Leow, C.Y., Nour Hindia, M.H.D. (2018). An overview of Internet of Things (IoT) and data analytics in agriculture: Benefits and challenges. IEEE Internet of Things Journal, 5(5): 3758-3773. https://doi.org/10.1109/JIOT.2018.2844296

[17] Petru, M., Novák, O., Lepšík, P. (2014). Analysis and measurement of the charge intensity of the selected electrospinning electrodes. Applied Mechanics and Materials, 486: 217-222. https://doi.org/10.4028/www.scientific.net/AMM.486.2 17

[18] Minh, V.T., Moezzi R., Owe I. (2018). Fuel economy regression analyses for hybrid electric vehicle. European Journal of Electrical Engineering, 20(3): 363-377. https://doi.org/10.3166/EJEE.20.363-377

[19] Hana, P., Inneman, A., Daniel, V., Sieger, L., Petru, M. (2015). Mechanical properties of carbon fiber composites for applications in space. Paper presented at the Proceedings of SPIE - the International Society for Optical Engineering, pp. 94420A. https://doi.org/10.1117/12.2175925

[20] Prathibha, S.R., Hongal, A., Jyothi, M.P. (2017). IoT based monitoring system in smart agriculture. In 2017 International Conference on Recent Advances in Electronics and Communication Technology, pp. 81-84. https://doi.org/10.1109/icraect.2017.52

[21] Cyrus, J., Krcmarik, D., Moezzi, R., Koci, J., Petru, M. (2019). Hololens used for precise position tracking of the third party devices - autonomous vehicles. Communications - Scientific Letters of the University of Zilina, 21(2): 18-23. 